\begin{tabular}{|c|c|}
\hline Title: & $\begin{array}{l}\text { Measurement of Two-Level Inverter Induced Current Slopes at High Switching } \\
\text { Frequencies for Control and Identification Algorithms of Electrical Machines }\end{array}$ \\
\hline Authors: & Simon Decker, Andreas Liske, Daniel Schweiker, Johannes Kolb, Michael Braun \\
\hline Institute: & $\begin{array}{l}\text { Karlsruhe Institute of Technology (KIT) } \\
\text { Elektrotechnisches Institut (ETI) }\end{array}$ \\
\hline Type: & Conference Proceedings \\
\hline Published at: & $\begin{array}{l}\text { Proceedings } 2018 \text { International Power Electronics Conference (IPEC-Niigata } 2018 \text { - } \\
\text { ECCE Asia), Niigata, Japan, 20-24 May } 2018 \\
\text { Publisher: IEEE } \\
\text { Year: } 2018 \\
\text { ISBN: } 978-4-88686-405-5\end{array}$ \\
\hline Hyperlinks: & 10.23919/IPEC.2018.8507431 \\
\hline
\end{tabular}

(C) 2018 IEEE. Personal use of this material is permitted. Permission from IEEE must be obtained for all other uses, in any current or future media, including reprinting/republishing this material for advertising or promotional purposes, creating new collective works, for resale or redistribution to servers or lists, or reuse of any copyrighted component of this work in other works. 


\title{
Measurement of Two-Level Inverter Induced Current Slopes at High Switching Frequencies for Control and Identification Algorithms of Electrical Machines
}

\author{
Simon Decker ${ }^{1 *}$, Andreas Liske ${ }^{1}$, Daniel Schweiker ${ }^{1}$, Johannes Kolb ${ }^{2}$ and Michael Braun ${ }^{1}$ \\ 1 Institute of Electrical Engineering, Karlsruhe Institute of Technology, Karlsruhe, Germany \\ 2 SHARE at KIT, Schaeffler Technologies AG \& Co. KG, Karlsruhe, Germany \\ *E-mail: simon.decker@kit.edu
}

\begin{abstract}
Several modern control and online identification algorithms for electrical machines are based on fast current slope detection. This paper shows and compares several identification methods for the inverter induced current slopes at high switching frequencies and high bandwidth of the measured signal. Test bench measurements with a SiC-MOSFET-inverter with switching frequencies up to $60 \mathrm{kHz}$ and an RL-load are used to compare the different identification methods. Best results among the investigated methods have been achieved with an easy implementable printed circuit board (PCB) design of a planar Rogowski coil.
\end{abstract}

Keywords - current slope measurement, online parameter identification, PCB integrated planar Rogowski coil, sensorless control.

\section{INTRODUCTION}

Precise and fast identification of the inverter induced current slopes is mandatory for many sensorless or selfsensing control schemes [1], adaptive control algorithms [2], online parameter identification [3], rotor-temperature identification [4] or inner fault detection [5] of electrical machines.

Higher switching frequencies of actual silicon-carbide semiconductors are desired to reduce weight and space and increase power density compared to the regular IGBT inverters. This requires a higher bandwidth for the measurement- and signal processing equipment. Especially the requirements for the fast current slope identification are increasing.

This paper describes and compares some measurement principles and sensors for fast current slope identification at high switching frequencies for future power electronic devices and control algorithms.

Section II. of this paper gives an overview of the different implemented identification methods. The next chapters discusses the implementation of the investigated methods in hard- and software. Section V. shows the experimental setup of the power electronics and signal-processing test- bench hardware. Then the evaluation and comparison of measurements results of the different identification methods are illustrated in section VI. In the last section a short summary is given.

\section{CURRENT Slope SENSING}

Current slope detection can be done with several methods, which can be categorized into direct and indirect current slope identification.

Direct current slope identification means direct sensing of the current slopes ahead the analog-digital converter. This can be realized e.g. with a hardware circuit which differentiates the absolute sensor signal of a current transducer, as proposed in [6]. Another possibility is the use of Rogowski coil based current transducers which has the advantage of high bandwidth. But the disadvantage is the necessary integrator circuit for absolute current measurement [7]. By using Faradays law of induction the Rogowski coil yields direct to a $\frac{\mathrm{d} i}{\mathrm{~d} t}$ value, which has to be integrated to get the absolute current value. Without the integration, only the raw current slope can be measured and evaluated.

Indirect current slope identification means, that the current slopes are calculated in the signal-processing hardware after the analog digital conversion. This is done by evaluating the measured absolute current values for example with least-squares estimators [2], linear regression or current deviation principles [1].

The absolute current measurement values can be determined with different physical principles. Ohm's law can be evaluated by shunt resistors, Faradays law of induction is used e.g. in current transformers. Magnetic field sensors based on the Hall-effect or the Fluxgate principle as well as magneto resistive effects like the Anisotropic Magneto Resistance (AMR). Several other principles like Faraday effect sensors, etc. are described in [8]. 


\section{HARDWARE IMPLEMENTATION}

Galvanic insulated current sensors are typical for power electronic applications. Hence, these sensors are used to compare the different current slope identification methods. Typical sensors for power electronic applications are magnetic field sensors like Hall-effect sensors and magneto resistive effect sensors like AMR sensors. Therefore the Hall-effect sensor LAH 25-NP from LEM and the AMR sensor CMS3025 from Sensitec are chosen for the indirect current slope identification methods. In Tab. I. both sensor characteristics are listed.

TABLE I

INVESTIGATED CURRENT SENSORS FOR INDIRECT CURRENT SLOPE DETECTION

\begin{tabular}{ccc}
\hline & Hall-effect sensor & AMR sensor \\
\hline Model & LAH $25-\mathrm{NP}$ & $\mathrm{CMS} 3025$ \\
Rated current & $25 \mathrm{~A}$ & $25 \mathrm{~A}$ \\
Bandwidth & $200 \mathrm{kHz}$ & $2 \mathrm{MHz}$ \\
Delay & $0.5 \mu \mathrm{s}$ & $0.06 \mu \mathrm{s}$ \\
Accuracy & $\pm 0.3 \%$ & $\pm 1 \%$ \\
Sensing & $i(t)$ & $i(t)$ \\
Sensitivity & $1 \frac{\mathrm{mA}}{\mathrm{A}}$ & $40 \frac{\mathrm{mV}}{\mathrm{A}}$ \\
\hline
\end{tabular}

The in Tab. I. listed sensors are mounted on a PCB and provided with filters according to the reference design from the manufacturer. The current sensor signal of the LAH 25-NP is converted to a voltage signal with a $100 \Omega$ measuring resistor, and amplified to $\pm 10 \mathrm{~V}$ at $\pm 50 \mathrm{~A}$ for the signal-processing hardware.

The CMS3025 voltage output signal is directly amplified to $\pm 10 \mathrm{~V}$ at $\pm 50 \mathrm{~A}$.

For the direct measurements an analog differentiator circuit is implemented, according to [6]. A planar Rogowski coil for a PCB is designed, based on the "pick up coil" as described in [9]. Additionally a prototype $\mathrm{d} i / \mathrm{d} t$ sensor for direct current slope measurement from Sensitec, based on a Rogowski coil, is investigated. In Tab. II. the investigated sensors are listed.

TABLE II

INVESTIGATED AND DEVELOPED CURRENT SLOPE SENSORS FOR DIRECT CURRENT SLOPE DETECTION

\begin{tabular}{cccc}
\hline & di/d $t$-sensor & $\begin{array}{c}\text { Planar } \\
\text { Rogowski } \\
\text { coil }\end{array}$ & $\begin{array}{c}\text { Analog } \\
\text { Differentiator }\end{array}$ \\
\hline Model & SENSITEC & PROTOTYPE & PROTOTYPE \\
Rated & $25 \mathrm{~A}$ & - & - \\
current & n.a. & n.a. & $100 \mathrm{kHz}$ \\
Bandwidth & n.a. & n.a. & n.a. \\
Accuracy & n.a. & n.a. & n.a. \\
Sensing & $\frac{\mathrm{d} i}{\mathrm{~d} t}$ & $\frac{\mathrm{d} i}{\mathrm{~d} t}$ & $\frac{\mathrm{d} v}{\mathrm{~d} t}$ \\
Sensitivity & $41 \frac{\mathrm{mV}}{\mathrm{kA} / \mathrm{s}}$ & $0.6 \frac{\mathrm{mV}}{\mathrm{kA} / \mathrm{s}}$ & $\mathrm{n} . \mathrm{a}$. \\
\hline
\end{tabular}

The Sensitec $\mathrm{d} i / \mathrm{d} t$-sensor Prototype is evolved from a CMS3025 current sensor with an additional output for the current slope signal. The signal is generated by a Rogowski coil mounted opposite to the busbar followed by an integrated amplifier.

In [9] and [10] a planar Rogowski coil for current sensing and its implementation is introduced. The planar Rogowski coil is integrated in the top and bottom layer of the PCB and connected through vias. The conductor is an isolated external mounted busbar soldered on the top layer. The top and side view of the design is shown in Fig. 1 and Fig. 2. The busbar is made of copper with a thickness of $0.5 \mathrm{~mm}$ and a distance $z_{0}=0.25 \mathrm{~mm}$ to the planar Rogowski coil.

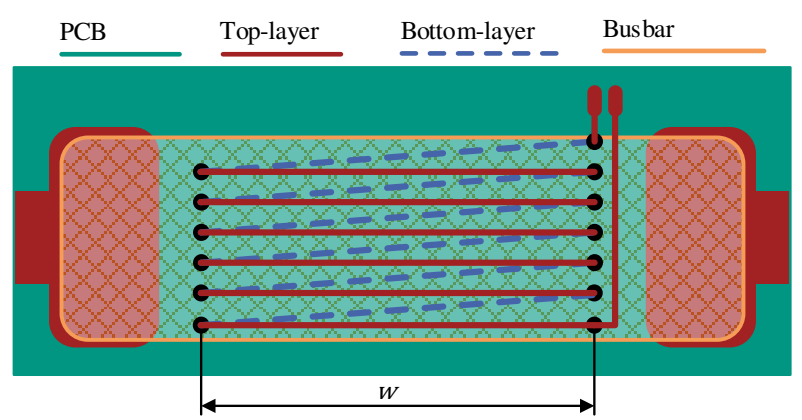

Fig. 1. Top view of the PCB integrated planar Rogowski coil for one of the three-phases.

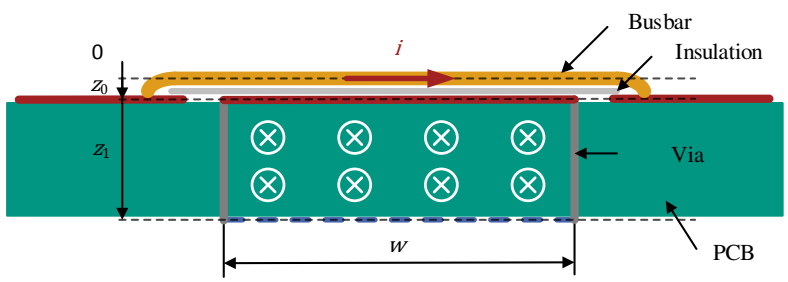

Fig. 2. Side view of the PCB integrated planar Rogowski coil for one of the three-phases.

The flux density $B$ induced in the sensor coil by the busbar can be calculated according to equation (1).

$$
\vec{B}(r)=\mu_{0} \frac{i}{2 \pi r} \cdot \vec{e}_{\varphi}
$$

Assuming a homogenous field of the conductor, the flux inside the planar Rogowski coil can be determined with equation (2).

$$
\Phi=\int_{z_{0}}^{z_{1}} \mu_{0} \frac{i \cdot w}{2 \pi r} \mathrm{~d} r=\mu_{0} \frac{i \cdot w}{2 \pi} \cdot \ln \left(\frac{z_{1}}{z_{0}}\right)
$$


With Faraday's law of induction the induced voltage $v(t)$ defined in equation (3) can be derived to the mutualinductance $M$ multiplied with the current slope $\frac{\mathrm{d} i}{\mathrm{~d} t} . N$ is thereby the number of turns, $w=50 \mathrm{~mm}$ the width of the planar Rogowski coil.

$$
v(t)=N \cdot \frac{\mathrm{d} \Phi}{\mathrm{d} t}=M \cdot \frac{\mathrm{d} i}{\mathrm{~d} t}
$$

The self-inductance, significant for the dynamic behavior, is analytically calculated with Niwa's method for rectangular coil inductances [11].

The required sensitivity for equal conditions between the compared direct current slope identification methods is thereby adopted from the $\mathrm{d} i / \mathrm{d} t$-sensor to $41 \frac{\mathrm{mV}}{\mathrm{kA} / \mathrm{s}}$. The planar Rogowski design sensitivity has to be amplified with an operational amplifier circuit to the required sensitivity.

The analog differentiator circuit is based on a design presented in [6] and can be assembled to the current sensors. The circuit is shown in

Fig. 3. It consists of a passive, first order high-pass filter and a two-stage operational amplifier (op-amp) circuit. The high speed op-amps are ADA49898-1 from Analog Devices. The input voltage $v_{\text {in }}$ is generated by the current transducer from the absolute measured value. The output voltage $v_{\text {out }}$ is described with equation (4).

The response time $\tau=R_{1} C_{1}$, directly influences the dynamics of the voltage $v_{\text {out }}$. Hence, for the dimensioning of $\tau$, the switching frequency of the inverter has to be taken into account. It is assumed that the final value of $v_{\text {out }}$ is reached after $3 \tau$. The time constant $\tau$ is set to $\tau=\frac{0.02}{3 f}$, for a minimum pulse-width of $\frac{0.02}{f}$ to be able to measure the current slopes at small duty cycles. This has to represent a duty cycle of about $2 \%$.

$$
v_{\text {out }}=\left(R_{1} C_{1} \frac{\mathrm{d} v_{\text {in }}}{\mathrm{d} t}\right) \cdot\left(1+\frac{R_{21}}{R_{22}}\right) \cdot\left(1+\frac{R_{31}}{R_{32}}\right)
$$

Adding the capacitors $C_{2 \mathrm{x}}, C_{3 \mathrm{x}}$ results in a low-pass filter and reduces the noise at higher frequencies.

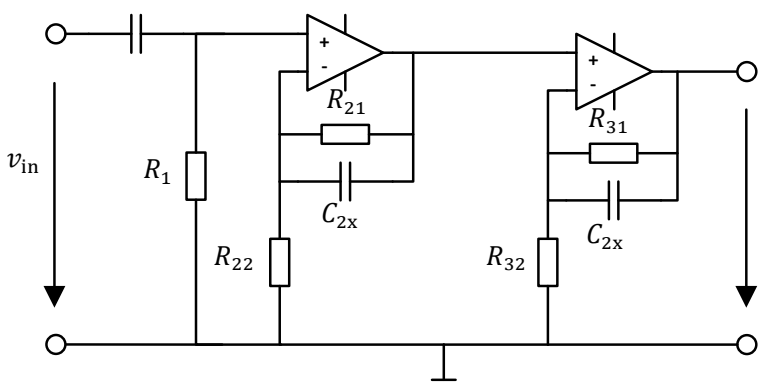

Fig. 3. Schematic of the analog differentiator circuit.

\section{SOFTWARE IMPLEMENTATION}

The signal processing system calculates the necessary duty cycles for a given sine wave form which are the reference values for the measurement.

Several methods of current slope evaluations are described in the literature. Common methods are e.g. moving average or least-squares algorithms, artificial neuronal networks and several other principles for software-based current slope identification [12].

In this paper an easy software-based identification method for the current slope will be introduced and discussed. The inverter induced current ripple depends on the switching states of the pulse width modulation. With the knowledge of the applied switching states and the assumption of linearly rising and falling currents between those, the current slopes can be calculated. Two measured current values and the time between these, are sufficient. This in principle, is similar to the described method in [1]. Both necessary current values are generated from the average value of a sequence of oversampled current samples during the switching state. Due to the averaging for the two measured current values, a better signal to noise ratio is achieved especially at higher duty cycles. Samples close to the switching transitions are neglected in the calculation. In Fig. 4 the current slopes and the switching states are displayed. The blue and black circles and the green squares mark the oversampling current. The current slope of III. is calculated with the average value, marked with a star, of the sequence of the blue circles and the average value of the sequence of the green squares. The difference $\Delta \bar{I}$ among the current values and the time $\Delta t$ between these yields to the current slope during this switching state. This procedure is repeated each switching state.

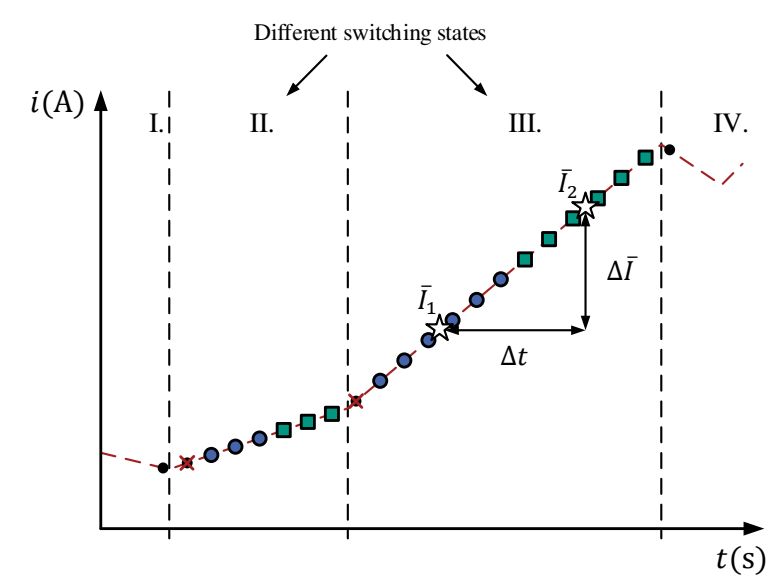

Fig. 4. Principle of the sequential current slope calculation with oversampled absolute current values. 


\section{EXPERIMENTAL SETUP}

The single PCB, Silicon-Carbide MOSFET voltage source inverter consists of a CCS050M12CM2 Six-Pack Modul from Wolfspeed, the DC-link capacitors, the current transducers CMS3050 from Sensitec, an internal fault management and protection system. The inverter switching frequency is up to $100 \mathrm{kHz}$. The calculation of the reference values and the evaluation of the AnalogDigital Converters (ADCs) is implemented on a real-time system based on a Xilinx Zynq-7030 System on Chip (SoC) with a dual-core ARM Cortex A9 processor and a Kintex-7 Field-programmable gate array (FPGA).

The measured current values are sampled with a 15-bit and five Mega samples per second (Msps) ADC from Linear Technology LTC2323-14. For reference measurements the Agilent DSOX3024A oscilloscope with $200 \mathrm{MHz}$ bandwidth and the Agilent current probe 1147 A are used. The RL-load is a power yoke with shared iron core which has an inductance of $230 \mu \mathrm{H}$ and a resistance of $300 \mathrm{~m} \Omega$.

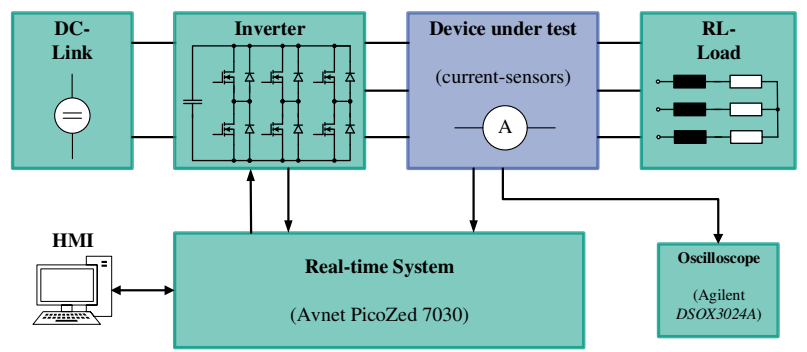

Fig. 5. Experimental setup of the test-bench. The real-time system needs measurement of the currents for the software based evaluation of the current slopes and the control of the RL-load.

\section{MEASUREMENT RESULTS}

The measurement results in this section are used for a qualitative analysis of the investigated current slope identification methods. The measured time variant current slope signals can thereby compared to each other with regard to control and identification methods usability.

For the measurements a fundamental sine wave current with an RMS value of $25 \mathrm{~A}$ and a frequency $f_{0}=450 \mathrm{~Hz}$ is generated with a DC-link voltage of $80 \mathrm{~V}$. The switching frequency of the two-level inverter for the first measurement is $10 \mathrm{kHz}$, for the second measurement $60 \mathrm{kHz}$. For the evaluation of the investigated current sensors, the outputs from the LEM LAH 25-NP and the Sensitec CMS3025 are compared with a current probe from Agilent. In the first measurement with $10 \mathrm{kHz}$ switching frequency, the signals of the current transducers are similar and not displayed this paper. In the second measurement (Fig. 6.) at $60 \mathrm{kHz}$ the delay in the signal from the LAH 25-NP sensor is clearly visible due to the limited bandwidth. The signal from the CMS3025 conform to the signal from the current probe from Agilent. The signal from the Agilent current probe is displayed black, the one from the CMS3025 sensor red and the LAH 25-NP sensor green.

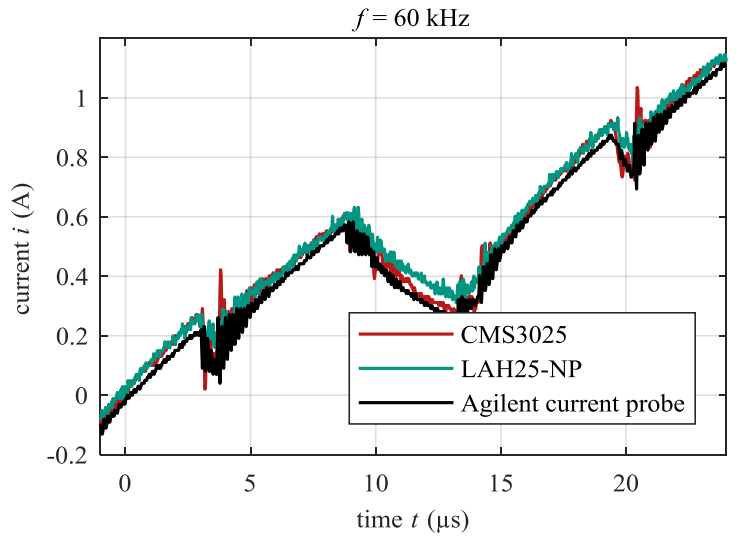

Fig. 6. Reference measurement of the absolute value of the investigated current sensors compared to the current probe.

In the following subsections the current slope detection methods measurements are discussed. In each section first the measurement with $10 \mathrm{kHz}$ switching frequency is displayed, followed by the measurement with $60 \mathrm{kHz}$ switching frequency. Thereby the grey current slope reference signal is generated from the absolute measured values of the current sensors which are logged by the oscilloscope and derived afterwards. In each figure also the measured current value is shown. The signal form of the investigated current slope detection sensor/method is displayed in blue.

\section{A. di/dt-sensor}
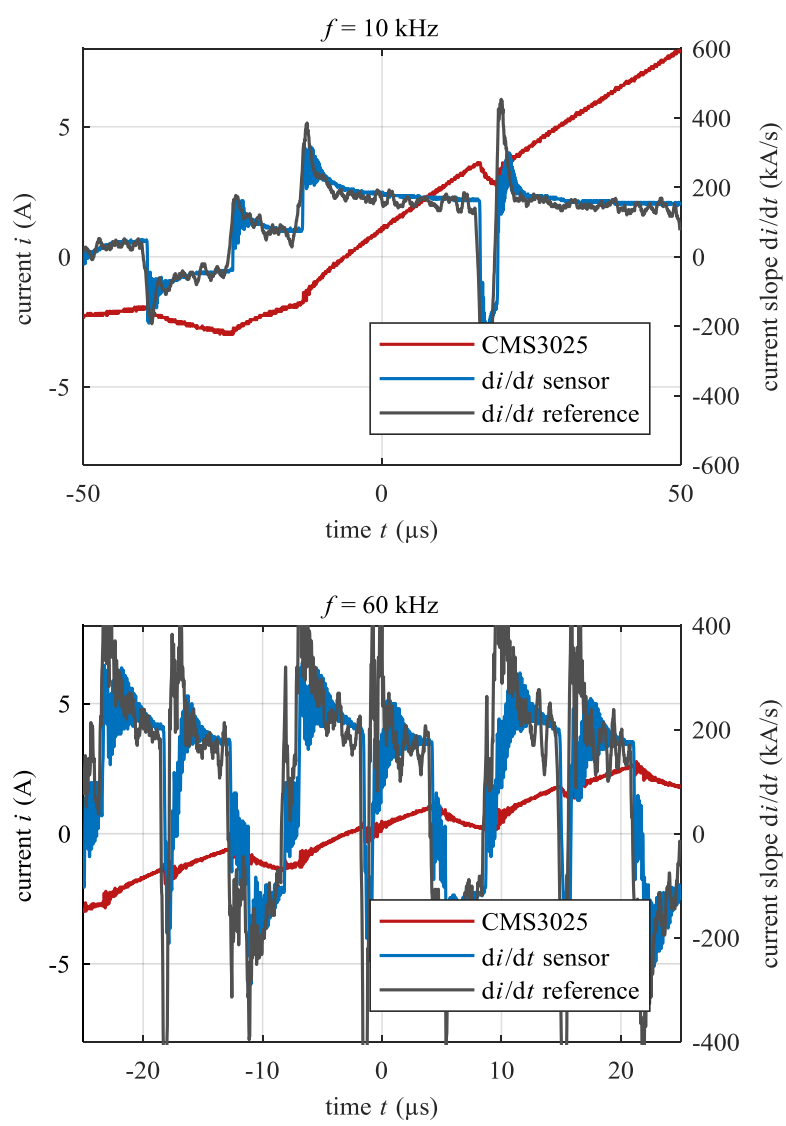

Fig. 7. Measurement of the $\mathrm{d} i / \mathrm{d} t$-sensor prototype with $10 \mathrm{kHz}$ and $60 \mathrm{kHz}$ switching frequency. 
At $10 \mathrm{kHz}$ the $\mathrm{d} i / \mathrm{d} t$-sensor in blue shows the expected waveform. At small pulse width of approximately $15 \mu \mathrm{s}$ a delay is visible. But due to the switching transitions these detected current slopes of these small duty cycles cannot be evaluated anyway.

At $60 \mathrm{kHz}$ the signal is of high noise and the sensor shows delays. The current slopes at small duty cycles cannot be measured due to the limited bandwidth probably because of the high self-inductance of the used Rogowski coil. But the reference value in gray is noisy as well due to the calculation of the derivative from the measured absolute value of the CMS3025 sensor.

\section{B. PCB planar Rogowski coil}
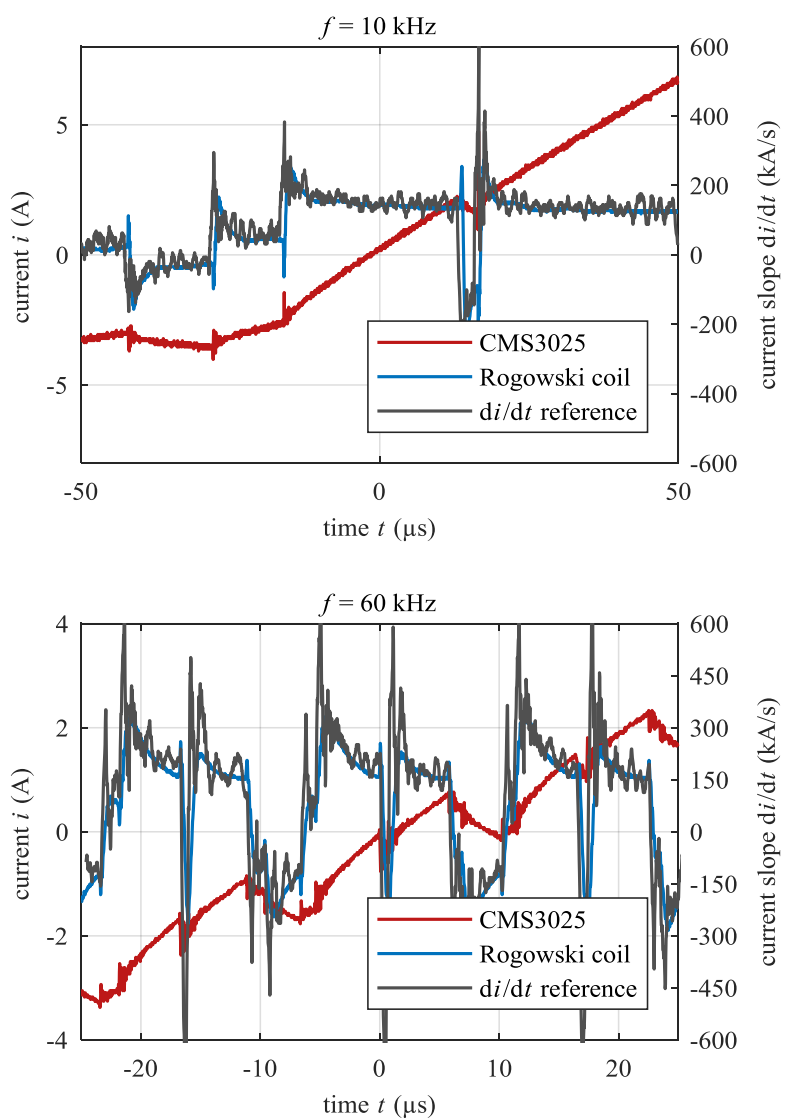

Fig. 8. Measurement of the self-designed planar PCB Rogowski coil prototype with $10 \mathrm{kHz}$ and $60 \mathrm{kHz}$ switching frequency.

The PCB integrated planar Rogowski coil delivers better results compared to the current slope reference measurement, generated from the CMS3025 signal in red. Small delays at small duty cycle indicate a limited bandwidth, but even at these small duty cycle the average current slope is identified precise and correctly.

\section{Analog differentiator}
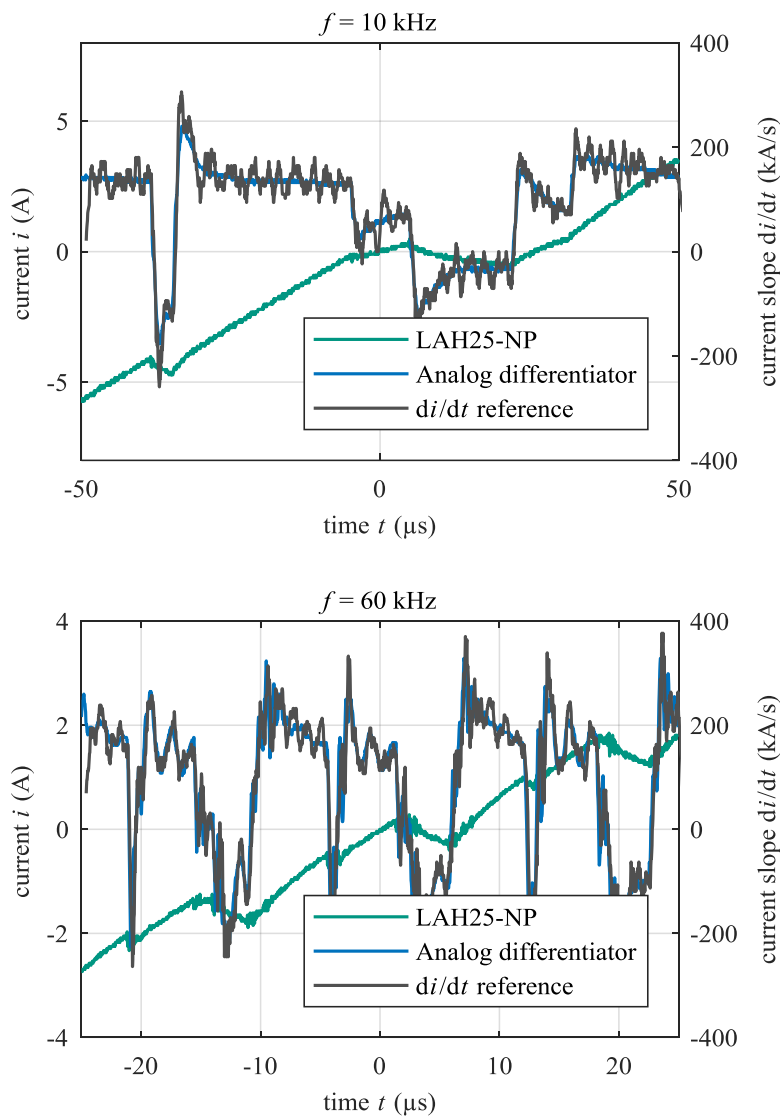

Fig. 9. Measurement of the analog differentiator circuit in combination with the LAH 25-NP with $10 \mathrm{kHz}$ and $60 \mathrm{kHz}$ switching frequency.

The CMS3025 sensor in combination with the designed analog differentiator circuit did not lead to good results. Due to the high bandwidth of the CMS3025 the circuit was too sensitive for valid results. Therefore, the LAH 25-NP signal with a lower bandwidth was evaluated with the designed analog differentiator circuit. The quality of the analog differentiator circuit output depends thereby on the signal quality from the LAH 25-NP sensor.

\section{Software current slope identification}

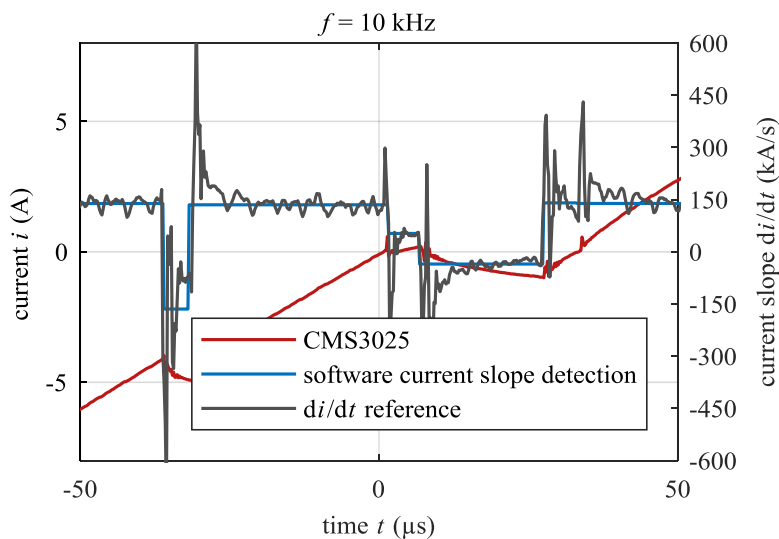

Fig. 10. Measurement of the proposed software based current slope detection with $10 \mathrm{kHz}$ switching frequency. 
The online calculation of the actual current slope is processed on the introduced real-time systems programmable logic. Therefore the measured value from the CMS3025 current sensor is digitized and evaluated with the presented algorithm above.

The mean value of the current slope of the $10 \mathrm{kHz}$ switching frequency measurement is thereby identified correctly, seen in Fig. 10. However small duty cycles cannot be evaluated correctly, because of the limited bandwidth of the AD-conversion. The second measurement with $60 \mathrm{kHz}$ is not evaluated due to the limited bandwidth and is not displayed here.

\section{CONCLUSION}

This paper investigates different identification methods for the inverter induced current slope. The principles of different methods for current slope detection are explained. The design of an analog differentiator, a planar PCB-integrated Rogowski coil and a software based current slope identification is shown. The presented techniques are implemented on a test-bench and compared to each other. Additionally a $\mathrm{d} i / \mathrm{d} t$-sensor prototype is used for parallel measurement. Every tested sensor and principle work well so far for standard two level inverters with a switching frequency below $10 \mathrm{kHz}$. The quality of the output signal of the analog differentiator depends thereby highly on the input signal. Increasing the switching frequency up to $60 \mathrm{kHz}$ leads to high-noise signals and increases the requirements of filter circuits and EMC of all sensors. The developed and designed simple and cheap planar Rogowski coil offers the best signals at these high switching frequencies.

\section{REFERENCES}

[1] E. Robeischl and M. Schroedl, "Optimized INFORM Measurement Sequence for Sensorless PM Synchronous Motor Drives With Respect to Minimum Current Distortion," in IEEE Transactions on Industry Applications, 2004, pp. 591-598.

[2] A. Liske and M. Braun, "Direct adaptive current control a universal current control scheme for electrical machines," in Industrial Electronics Society, IECON 2014 - 40th Annual Conference of the IEEE, 2014, pp. 514-520.

[3] S. Decker, J. Richter, and M. Braun, "Predictive current control and online parameter identification of interior permanent magnet synchronous machines," in 18th European Conference on Power Electronics and Applications (EPE'16 ECCE Europe), 2016, pp. $1-10$.

[4] M. Ganchev, C. Kral, and T. Wolbank, "Identification of sensorless rotor temperature estimation technique for Permanent Magnet Synchronous Motor," in International Symposium on Power Electronics Power Electronics, Electrical Drives, Automation and Motion, 2012, pp. 38-43.

[5] T. M. Wolbank, P. Nussbaumer, H. Chen, and P. E. Macheiner, "Non-invasive detection of rotor cage faults in inverter fed induction machines at no load and low speed," in IEEE International Symposium on Diagnostics for Electric Machines, Power Electronics and Drives, 2009, pp. 1-7.

[6] P.-H. Liu, Y. Yan, F. C. Lee, and P. Mattavelli, "External ramp autotuning for current mode control of switching converters," in Twenty-Eighth Annual IEEE Applied Power Electronics Conference and Exposition (APEC), 2013, pp. 276-280.
[7] Texas Instruments Incorporated, High Accuracy AC Current Measurement Reference Design Using PCB Rogowski Coil Sensor (Rev. A). [Online] Available: http://www.ti.com/tool/TIDA-01063.

[8] S. Ziegler, R. C. Woodward, H. H.-C. Iu, and L. J. Borle, "Current Sensing Techniques: A Review," in IEEE Sensors Journal, 2009, pp. 354-376.

[9] L. Zhao, J. D. van Wyk, and W. G. Odendaal, "Planar embedded pick-up coil sensor for integrated power electronic modules," in Applied Power Electronics Conference and Exposition, 2004. APEC '04. Nineteenth Annual IEEE, 2001, pp. 945-951.

[10] Y. Xue et al., "A compact planar Rogowski coil current sensor for active current balancing of parallel-connected Silicon Carbide MOSFETs," in 2014 IEEE Energy Conversion Congress and Exposition (ECCE), 2014, pp. 4685-4690.

[11] F. Grover, Inductance calculations: Working formulas and tables. Research Triangle Park: Instrument Society of America, 19821946.

[12] D. Hind, C. Li, M. Sumner, and C. Gerada, "Realising robust low speed sensorless PMSM control using current derivatives obtained from standard current sensors," in 2017 IEEE International Electric Machines and Drives Conference (IEMDC), 2017, pp. 1-6. 